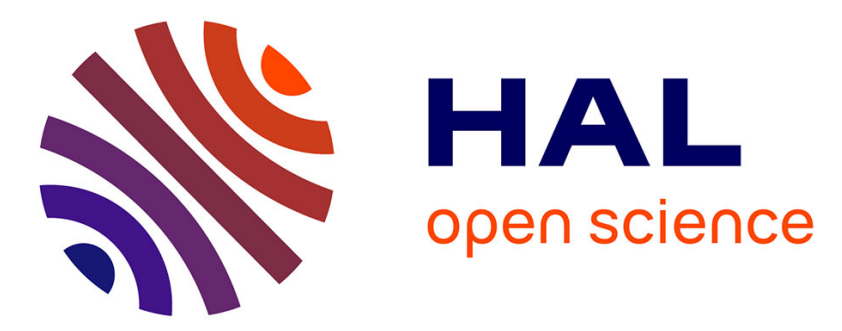

\title{
Four dimensional wave propagation simulation in liquid metal with realistic thermal fluctuation
}

Masaru Nagaso, Joseph Moysan, Christian Lhuillier, Dimitri Komatitsch

\section{To cite this version:}

Masaru Nagaso, Joseph Moysan, Christian Lhuillier, Dimitri Komatitsch. Four dimensional wave propagation simulation in liquid metal with realistic thermal fluctuation. Ultrasonics, In press, 10.1016/j.ultras.2021.106528 . hal-03291833

\section{HAL Id: hal-03291833 \\ https://hal.science/hal-03291833}

Submitted on 19 Jul 2021

HAL is a multi-disciplinary open access archive for the deposit and dissemination of scientific research documents, whether they are published or not. The documents may come from teaching and research institutions in France or abroad, or from public or private research centers.
L'archive ouverte pluridisciplinaire HAL, est destinée au dépôt et à la diffusion de documents scientifiques de niveau recherche, publiés ou non, émanant des établissements d'enseignement et de recherche français ou étrangers, des laboratoires publics ou privés. 


\title{
Four dimensional wave propagation simulation in liquid metal with realistic thermal fluctuation
}

\author{
Masaru Nagaso ${ }^{\mathrm{a}, \mathrm{b}, *}$, Joseph Moysan ${ }^{\mathrm{a}}$, Christian Lhuillier ${ }^{\mathrm{b}}$, Dimitri \\ Komatitsch $^{\mathrm{a}, * *}$ \\ a Aix Marseille Univ, CNRS, Centrale Marseille, LMA UMR 7031, Marseille, France \\ ${ }^{b}$ CEA/DES/IRESNE/DTN/STCP/LISM, 13108 St Paul lez Durance, France
}

\begin{abstract}
In this paper, we performed a four-dimensional numerical simulation to calculate wave propagation in a thermal fluctuating liquid metal in order to obtain detailed knowledge on a wave propagation in coolant material of a Sodiumcooled Fast reactor (SFR). The wave and the medium are described in three spatial and one temporal dimensions. We made use of a massive data set to describe the fluctuating situation of the medium. This data set was provided by Computational Fluid Dynamics (CFD) with a Large-Eddy Simulation (LES) model, which calculated the temperature field with a higher spatial resolution than Reynolds-Averaged Navier-Stokes turbulence models (RANS). This data set was furthermore obtained from other studies on a numerical and physical experiment called PLAJEST that created mixing jets of liquid metal in order to simulate the status of running SFRs. Because of the limitation of computational resources, previous acoustic studies applied to such a medium could only use the spatial-temporally averaged fluctuating heterogeneity of a medium calculated by RANS turbulence model. This limitation may overlook wave fluctuation because of the difference of the resolution between computational fluid dynamics and acoustic wave length. Our numerical effort allowed us to study the most realistic acoustic wave propagation in liquid metal than in any former studies. A new

\footnotetext{
* Corresponding author

${ }^{* *}$ This paper is dedicated to our colleague, D. Komatitsch who passed away on 21 January

Email address: nagaso@lma.cnrs-mrs.fr (Masaru Nagaso)
} 2019.
\end{abstract}

Preprint submitted to Journal of $L^{A} T_{E} X$ Templates

July 19, 2021 
important result was obtained in this work as we demonstrated that ultrasonic measurements could follow thermal-hydraulic fluctuations in an opaque liquid with high sensitivity. This result was obtained through the definition of descriptors to analyse medium fluctuations along the wave path. We defined a very new measurement index, called hereafter Cumulated Temperature Fluctuation Intensity (CTFI), to correlate the variations in the thermal-hydraulic conditions to the wave variations. We demonstrated a good correlation between the second derivative of this index and the second derivative of several acoustic measurements, then we discussed the easiest measurements to be used in practice in an industrial setup.

Keywords: Ultrasound, Fluctuating liquid media, Non-Destructive Testing (NDT), numerical modeling, spectral finite elements (SEM), Large-Eddy

Simulation (LES)

2010 MSC: 00-01, 99-00

\section{Introduction}

The fourth generation of Sodium-cooled Fast Reactors (SFR) is actively developed within a framework of an international cooperative research project [1, 2]. In France, a research and development project of this fourth generation 5 SFR, so called ASTRID project, is now being carried out [3].

As SFRs use liquid sodium for their coolant material, which is completely opaque, the method to investigate the running state of SFRs is quite limited. Also because of the high reactivity of liquid sodium to air, it is important to reduce the number of mechanical parts inside of SFR design in order to reduce the risk of sodium leakage and the difficulty of maintenance. For those requirements, measurement methods using acoustic and elastic waves (i.e. non-destructive testing) are regarded as techniques which have advantages for monitoring SFRs [4, 5].

Before now, an acoustic method has been applied for the monitoring of the 15 position of subassembly heads. The so-called SONAR monitoring system was 
developed by the Alternative Energies and Atomic Energy Commission (CEA) 6]. Using a standard emitter-receiver setup, the SONAR system measures the distance between a target object and an acoustic probe thanks to the conversion of the round-trip time of flight. Several studies developed theoretical or experimental 2o approaches to improve ultrasonic telemetry. Wedges of objects could be used to create reflection points of interest. Lü et al. compared several high-frequency approximations by simulating acoustic scattering by an immersed rigid wedge. They confirmed that for a planar wedge, these approximations produce the same predictions [7, 8. They concluded that Physical Theory of Diffraction (PTD)

25 [9 more efficient as it requires integration only over the illuminated part of the scattering surface and they recommended it for implementation in modelling software. From a more practical issue, the modelling of such signals and its comparison with experiments is a key point to help designers to define better geometries for monitoring situations [10].

30

Acoustic method is also regarded as a powerful tool for temperature measurement of sodium coolant, because of its higher measurement frequency than thermo-couples [11].

Thus, acoustic measurement techniques have a great advantage for application to SFR monitoring but we still need to advance in the knowledge on wave 35 propagation in SFRs to increase the measurement accuracy and reliability, and to expand the application area of acoustic measurements. The need for advanced studies on wave propagation in SFRs comes from the complexity of the propagation medium status as acoustic characteristics of liquid sodium vary dynamically and in a complex manner during an SFR's operation. The aim of this study is to develop a new methodology for this monitoring that could be effective in any other liquid metals.

In the sodium coolant, the three fields, flow velocity field, temperature gradient field and gas bubble density field, may affect a wave propagation. In this study, we only focus on temperature gradient, as the effect from the flow ${ }_{45}$ velocity field is weaker than the effect of the temperature gradient field, in the case of an SFR's operation situation[12], and the temperature gradient may cause 
an undesirable inflection [13, 14]. The effect from the density of gas bubbles has been studied in [15, 16]. In our study, we considered that the density of gas bubbles is constant. The result of a recent study on the thermal-hydraulic state of SFRs [17] shows that the maximum variation rate of flow rate is about $4.5 \mathrm{~m} \mathrm{~s}^{-1}$ and the maximum variation rate of temperature may be about $23.4^{\circ} \mathrm{C}$ par $10 \mathrm{~cm}$. For SONAR application, the propagation distance is around $270 \mathrm{~mm}$. Ultrasonic telemetry in sodium for distance from $1 \mathrm{~m}$ to $5 \mathrm{~m}$ are sought or tested in various reactors [18, [19]. Thus this variation may be not negligible for the possible application of acoustic measurement in SFRs.

When considering the effect of temperature gradient, we need to take into account liquid sodium's temperature-dependent properties reported in [20]. This study shows that the density difference of liquid sodium with temperature change between the temperature range from the normal melting point to the normal boiling point may be calculated with the linear relation:

$$
\rho\left[\mathrm{kg} \mathrm{m}^{-3}\right]=1014-0.235 \cdot T[\mathrm{kelvin}],
$$

at normal atmospheric pressure, where $\rho$ is the density of sodium and $T$ is the sodium temperature in Kelvin degrees. The sound speed of liquid sodium decreases monotonically with temperature, which is caused by the decreasing of the inter-atomic interactions. In the range of the normal melting - boiling point $\left(371-1155^{\circ} \mathrm{K}\right)$, the sound speed in pure liquid sodium may be described based on a linear relation:

$$
\left.c_{p}\left[\mathrm{~m} \mathrm{~s}^{-1}\right]=2723-0.531 \cdot T \text { [kelvin }\right] .
$$

where $c_{p}$ is the celerity of ultrasonic waves in meters per second.

We have carried out several numerical studies on the wave propagation in order to advance our simulation method for liquid sodium. For an SFR's study, numerical calculation have great importance as the real size experiment of SFR 6o with actual liquid sodium is not possible, as there is no operating SFR for an experimental study. Thus a realistic modeling of wave propagation is essential for designing an acoustic measurement system for SFR application. 
In the former studies which we have conducted for researching a sodium temperature heterogeneity effect on a wave propagation, we have improved the modeling method of this heterogeneity. In the early stage, we applied stochastic methods which randomly generate a fluctuating temperature field using Gaussian Random process in [21, 22, 23, 24, 25]. This method has a strong advantage of not consuming a large amount of computer memory and time when generating a fluctuation with high resolution. However as remarked in [23], generated fields may not well model the geometrical heterogeneity, e.g. a mean flow field occurring due to an interaction with internal structures of SFR.

N. Massacret [13] applied a Computational Fluid Dynamics (CFD) calculation result with a Reynolds-Averaged Navier-Stokes (RANS) turbulent model. A RANS model requires relatively lower quantity of computational resources than 75 others, e.g. Direct Numerical Simulation (DNS) and Large-Eddy Simulation (LES), by filtering the small turbulences. Thus the spatial resolution is not enough when compared with the acoustic wavelength, and not suitable for verifying the acoustic effect from smaller fluctuation pattern.

In this study, we carried out three-dimensional numerical simulations with so application to a more realistic fluctuating propagation medium. In the SFRs, the thermal-hydraulic situation is generated by the sodium jets with a high temperature and surrounding sodium with a lower temperature, and mixing phenomena between them. As the modeling target of our study, we selected an experimental and numerical study called PLAJEST, since it targets the same object in the same condition, i.e. the upper-core region of an SFR in operation.

PLAJEST is a collaboration between the Japanese Atomic Energy Agency (JAEA), the U.S. Department of Energy (DOE), and by the Alternative Energies and Atomic Energy Commission (CEA). The main purpose of PLAJEST is to observe heat conduction at a liquid-solid boundary in a sodium cooling circuit, because the thermal fluctuations lead to high-frequency thermal fatigue and thus may cause cracks in adjoining structures of SFRs. The configuration of this experiment is shown in Figure 1 (a). After the experiment made by JAEA [26, 27], a thermal-hydraulic analysis was carried out by the CEA in its Service 
de Thermo-hydraulique et de Mécanique des Fluides (STMF) ([28], Figure 1

95 (b)). In that analysis, the results indicate a good fit on time-averaged normalized temperature and its fluctuation between experimental and numerical values.

We consider these CFD thermal-hydraulic computation results are well validated by the experiment and that allows us to process full-wave propagation numerical simulations in realistic conditions. CEA/STMF used a Large-Eddy

100 Simulation turbulence model for this simulation, thus we may utilize these temperature fields with higher spatio-temporal resolution. In the first part of this study we analyse the temperature field issued from the mixing jets and we show that it could be characterized by two representative points of the merging of jets.

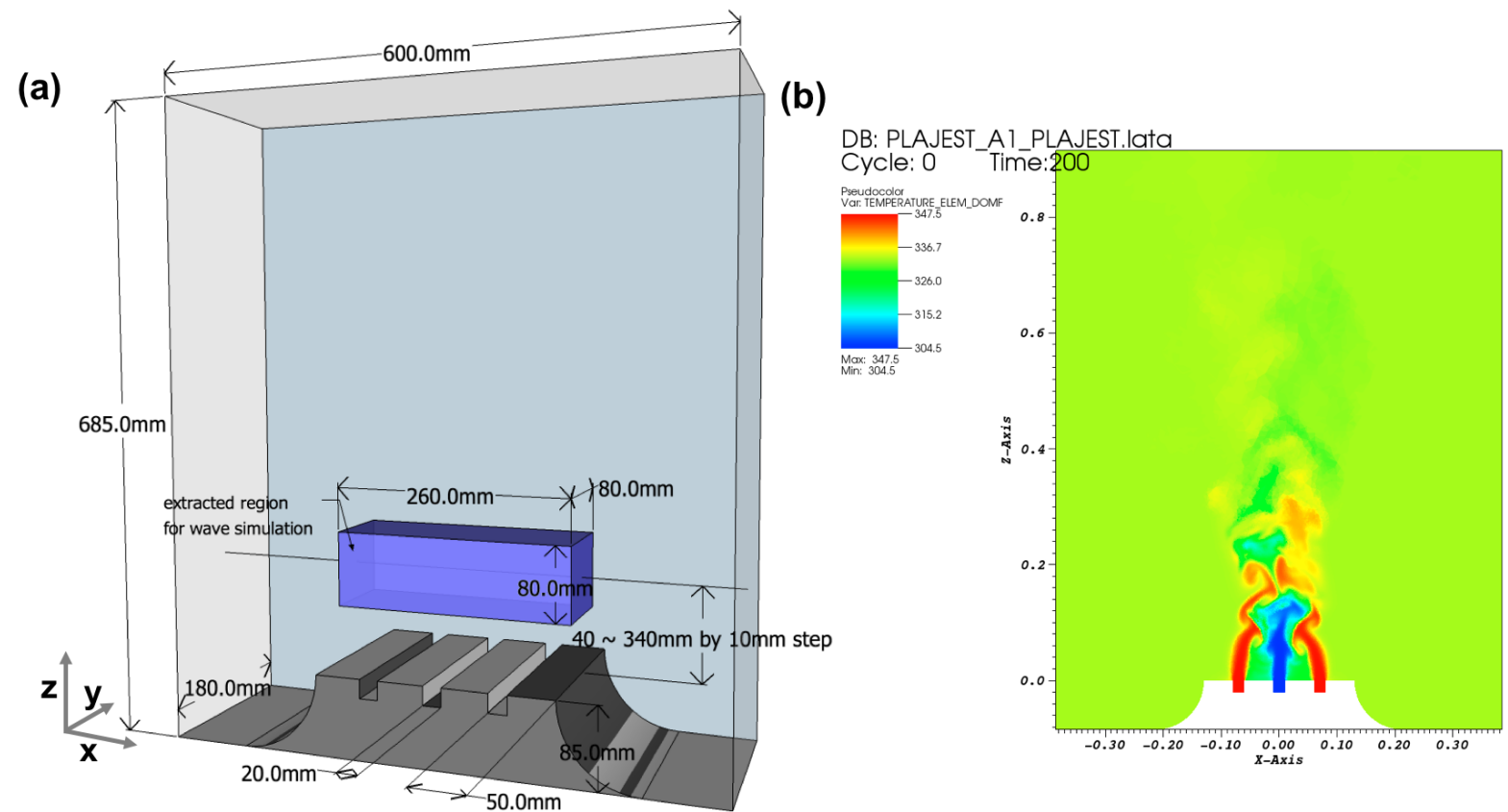

Figure 1: (a) Geometry of the PLAJEST CFD simulation. (b) Snapshot of the CFD result at time $200.0 \mathrm{~s}, x-y$ cross-sectional plane at $y=0.09 \mathrm{~m}$. Visualization for this image is done with VisIt, an open source visualization tool for massive scientific data [29].

In the second section, we firstly introduce the numerical method applied for wave propagation modeling and the technical details used for preparing 
the finite element mesh and the pre-processings for the CFD result data set. Then in the third section, we explain the method for analyzing the fluctuation of the thermal field and the acoustic field calculated with our model. In the fourth section, we show the details of the calculation setups used for our wave propagation calculations. We also show an example of the calculated 3D wave field propagating at one single moment of the fluctuating temperature field. In the fifth section, we detail the analysis method based on second derivative properties of several wave parameters. We demonstrate that ultrasonic measurements in 115 a through transmission configuration can find the two characteristic points of the temperature field. It opens the way to prospect industrial design to monitor such thermal-hydraulic situations using ultrasonic transducers.

\section{Numerical method}

\subsection{Spectral element method}

120

Our numerical wavefield modeling method is a time domain spectral-element method [30, which is a type of finite-element method using higher order basis functions (generally higher than 4th order) than an ordinal finite-element method using 1st or 2nd order basis functions.

We have chosen this method because this may model the heterogeneity of physical parameters, i.e. temperature and density values in our case, with higher resolution and lower number of elements, thus we may efficiently model the spacial domain of wave propagation. This method directly increases the limit of domain size and the frequency of modeled waves may becomes higher than the ordinal finite-element methods.

Following our previous studies [25, 31], we use the software SPECFEM3D [32] which is the one of the most well utilized softwares for full-wave wave propagation simulation because of its very matured and advanced development status especially for the use on HPC with the great numbers of CPUs [33] and GPUs [34, 35] as well. 
The CEA/STMF department used a numerical code for CFD calculations called TrioCFD (known as Trio_U by 2015) for this PLAJEST numerical simulation, and LES was selected as the turbulence model. Tetrahedral elements with 4 nodes were used for the TrioCFD calculations. The total number of elements was 5582706 and the characteristic mesh length was set to $1.40 \mathrm{~mm}$. We removed the first $200 \mathrm{~s}$ of their calculation from their result because that duration corresponds to the stabilization of the flow state. Thus, a time domain from 200 to $210 \mathrm{~s}$ with a time step of $0.1 \mathrm{~ms}$ is available for our wave simulation. Three jets of sodium exist in this setup. The sodium with lower temperature $\left(304.5^{\circ} \mathrm{C}\right)$ is emitted from the central jet, and the sodium with higher temperature $\left(347.5^{\circ} \mathrm{C}\right)$ is emitted from the two outer jets. The average flow velocity is $0.51 \mathrm{~m} \mathrm{~s}^{-1}$ for every jet. The simulated temperature field at time $=200.000 \mathrm{~s}$ is shown in Figure $1 \mathrm{~b}$. Their simulation results are in good agreement with the experiment results obtained by JAEA in terms of normalized time-averaged temperature, normalized time-averaged temperature fluctuations, spectral power density and standard deviation of temperature values.

For their calculation, a tetrahedral unstructured staggered mesh was used. Temperature field values are defined at the center of each TrioCFD's tetrahedral mesh element, and flow velocity values are defined on the vertex nodes. We thus had to transfer these values to our hexahedral mesh for SPECFEM3D. To do so, we used interpolation onto each node of the SPECFEM3D hexahedral mesh using the simulation data management tool called MEDCoupling. MEDCoupling is part of the pre-/post-processing platform SALOME (http://www.salome-platform. org) and is also available independently as a library. Figure 2 shows the temperature field data transfer and mesh generation steps as a pre-process for SPECFEM3D simulation. Our hexahedral mesh for SPECFEM3D is built using the meshing software CUBIT developed by Sandia National Laboratories (USA). It is possible to select an arbitrary volume to be extracted from the entire geometry and meshing is completed automatically, including the assignment of material characteristics and absorbing surface flags (the fluctuation of the 
temperature field may be defined later). After finishing preparation of mesh data, we carried out the temperature field transfer, i.e. interpolation of temperature values defined at the barycenter of each tetrahedral finite element to corner nodes of our hexahedral spectral elements. The flow velocity data is not used for our simulation because we apply the frozen fluid hypothesis, as in [12]. The conversion of the temperature field from TrioCFD tetrahedral mesh to SPECFEM3D nodes is done by using MEDCoupling.

Determination of the element size to use in our simulations is done based on two conditions, which are the CFL condition (Equation 3) and the number of elements per one wave length:

$$
C_{p} \frac{\Delta t}{\Delta x_{g l l}} \leq \alpha
$$

where $\Delta t$ is the time step and $\Delta x_{\text {gll }}$ is the minimum interval between two GaussLobatto-Legendre (GLL) grid points. GLL points are the additional collocation points inserted between the corner nodes of a general finite element. The position of those points are defined by numerically solving the equation:

$$
\left(1-x^{2}\right) P_{n}^{\prime}(x)=0
$$

where $P_{n}^{\prime}$ denotes the derivative of the $\mathrm{n}$-th order Legendre polynomial (i.e. $\left.\frac{d}{d x} P_{n}(x)\right)$. Then the recursion relation for these polynomials is

$$
P_{n+1}(x)=\frac{2 n+1}{n+1} x P_{n}(x)-\frac{n}{n+1} P_{n-1}(x), \quad P_{0}(x)=1, P_{1}(x)=x .
$$

Detailed explanations about GLL polynomials can be found in [36, 37].

We selected the averaged Courant number $\alpha=0.4$ and the wave celerity $C_{p}$ $175=2416.268 \mathrm{~m} \mathrm{~s}^{-1}$ (in sodium with the lowest temperature value $274.5^{\circ} \mathrm{C}$ in the CFD simulation) for the calculation of the mesh size and time step duration. In equation 3, $\Delta x_{g l l}$ is not the mesh size itself, it is the interval between GLL grid points inside the spectral elements. This led us to use a mesh size $\Delta x$ $=8.05 \times 10^{-4} \mathrm{~m}$ and a time step of $2.3 \times 10^{-8} \mathrm{~s}$. We simulate a total of 5000 steps in order to have a sufficient total physical duration for the waves to travel through the entire simulation domain. The mesh used in our simulations thus 3250000 spectral elements and a total number of GLL grid nodes of 215320764 . 
The figure 3 represents one of the heterogeneous temperature fields at one time step.

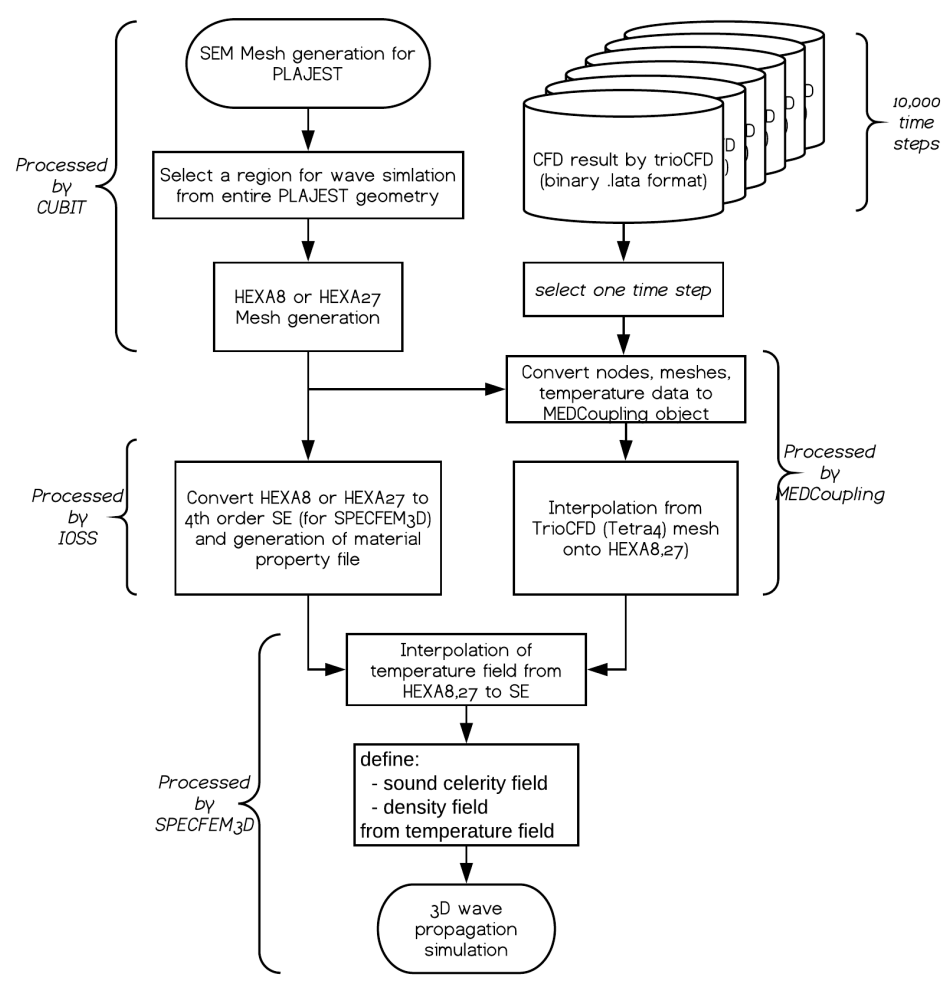

Figure 2: Explanation of data processing for mesh generation and preparation of the heterogeneous medium to use for our acoustic calculations.

\section{Medium fluctuation and acoustic fluctuation analysis}

The phenomenon of mixing flows has been actively studied by e.g. [38, [39] and [40]. These studies are not directly related to the PLAJEST experiment nor to liquid metal flows, but numerous thermal-hydraulic studies show that they have common thermal-hydraulic behaviors [14]. We therefore use the methodology proposed in these studies to analyze thermal-hydraulics. They identified the mixing state of flows and categorized them into three types based 


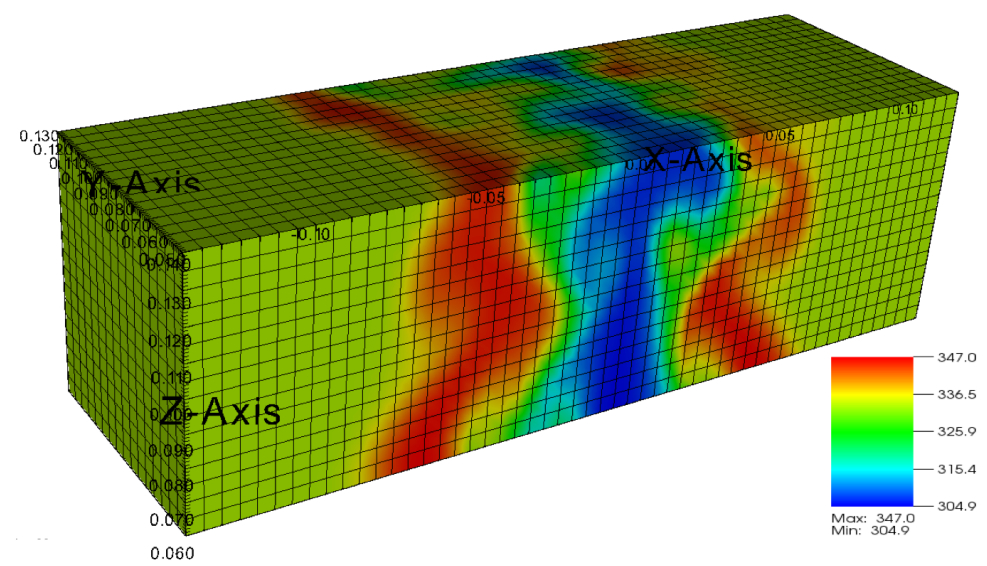

Figure 3: One of the heterogeneous temperature fields used for our 3D wave propagation calculations. This field is taken from time step number 10, and the central altitude of the calculation domain is $0.1 \mathrm{~m}$ from the sodium outlet. One mesh contains 3,250,000 spectral elements and the total number of GLL grid points is $215,320,764$.

on the state of the mean velocity, beginning from the bottom of the jets to the end of the jets. There is firstly a thermal-hydraulic region named the converging region, then exists the merging region, and at end the jets joined together in the combined flow region. The converging region starts at the exit of the flow and continues until the negative mean flow (i.e. the flow going in the opposite direction of the jets) disappears. The point where the negative flow disappears is called the merging point. At this merging point, each flow still conserves its own flow and they are not united yet. From the merging point, these flows start to gradually merge, and finally the mean flow distribution merges as one large flow. This point is called the combined point.

Durve and al. 38, also carried out a comparative study on several models for predicting the mean temperature field and temperature fluctuation field caused by mixing phenomena of the three jets [41].

205 Following different authors we choose to analyze the temperature in the medium using an index called the Temperature Fluctuation Intensity (TFI). We use the definition of TFI as 


$$
T F I(\boldsymbol{r})=\sqrt{\frac{1}{N} \sum_{i=1}^{N}(T(i, \boldsymbol{r})-\bar{T}(\boldsymbol{r}))^{2}},
$$

where $\boldsymbol{r}=(x, y, z)$ is the spatial position vector, $i$ is the time step number, $N$ is the total number of time steps, $T(i, \boldsymbol{r})$ is the temperature value at time 210 step $i$ and position $\boldsymbol{r}$, and $\bar{T}(\boldsymbol{r})=\frac{1}{N} \sum_{i=1}^{N} T(i, \boldsymbol{r})$ is the average temperature at $r$.

We process the PLAJEST Computational Fluid Dynamics (CFD) data to calculate the TFI index in order to compare the global behavior of the jets with these previous studies.

215 Figure 4 is the $2 \mathrm{D}$ cross-section at $y=0.09 \mathrm{~m}$ of the calculated $3 \mathrm{D}$ TFI field from the CFD results of PLAJEST. In this figure, a set of 1D TFI curves are superimposed to the $2 \mathrm{D}$ cross-section TFI image, which are calculated at several altitudes. There are three jets in the configuration of PLAJEST (the center position of the jets are $x=-0.070 \mathrm{~m}, 0.0 \mathrm{~m}$ and $0.070 \mathrm{~m}$ ). Between each jet, two zones with high TFI value arise by the interaction of these jet flows. From this TFI field, it is found that from the shape of the TFI profile depending on the altitude, it seems to be possible to define three zones in a similar way as [41]. First, the two high TFI zones arise around altitude $z=0.05 \mathrm{~m}$ and these two zones are completely separated. Around altitude $z=0.08 \mathrm{~m}$ or lower altitude, the beginning of merging of the high TFI zones becomes clear (the lowest TFI value at point $b$ between the two peeks a and c starts to increase). Here there would be a specific point that we call a merging point of the TFI zone. The merging of these two zones is confirmed when the altitude becomes higher, and then around altitude $z=0.18 \mathrm{~m}$ and $z=0.19 \mathrm{~m}$ these two zones are completely merged (the peaks a and c of the 1D TFI curve become a single one). We call this altitude a combining point of the TFI zone. These two altitudes are estimated of course on this numerical data.

In the following section, we carry out a more detailed analysis to verify the variations of the TFI profiles and the definition of these two points. The 


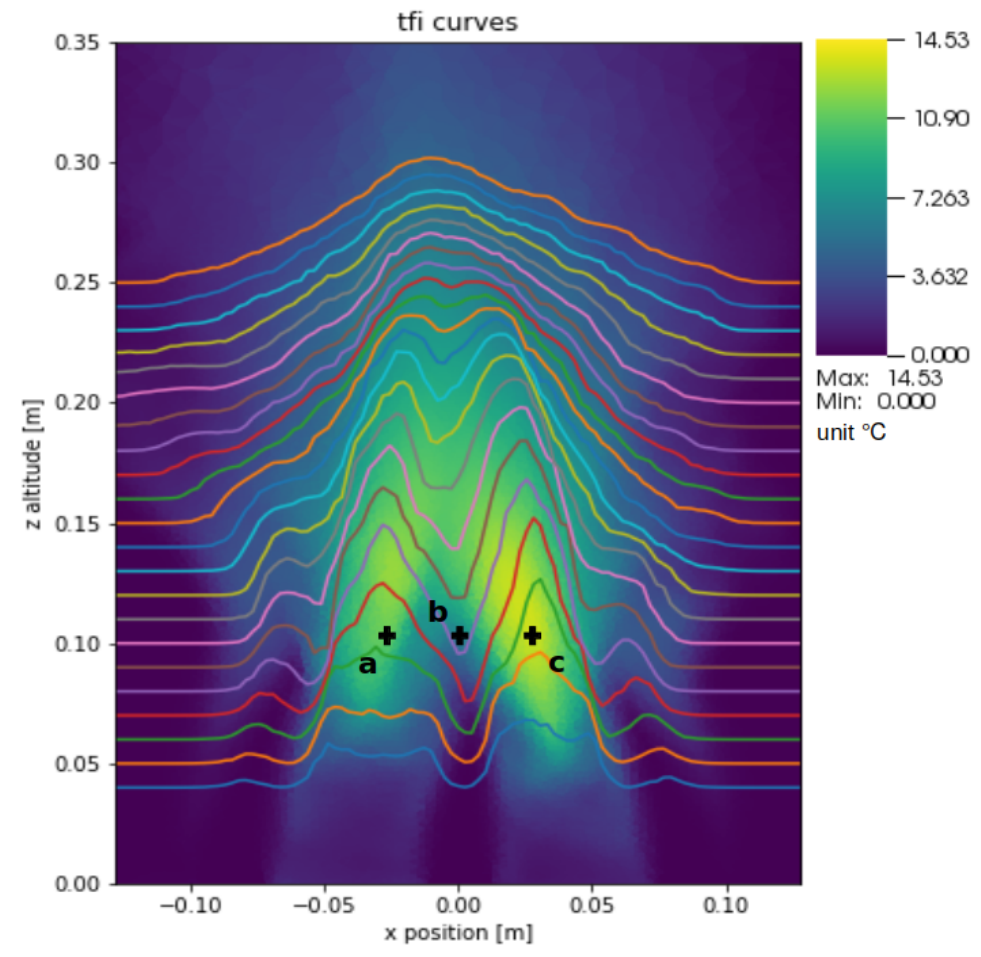

Figure 4: TFI field on a 2D cross-section at $y=0.09 \mathrm{~m}$ and $1 \mathrm{D}$ profiles. Each $1 \mathrm{D}$ profile is factored on vertical direction so that 0.1 on $\mathrm{z}$ altitude axis becomes the maximum TFI value for visualizing purpose. Points $\mathrm{a}, \mathrm{b}$, and $\mathrm{c}$ are the reference points used for $1 \mathrm{D}$ sequential analysis 


\subsection{Definition of the insonified volume}

The insonified volume is defined as a part of the PLAJEST volume displayed as a purple box in Figure 1 (a). In the geometry of PLAJEST, the three sodium jets outflow from gaps with a $20 \mathrm{~mm}$ width. Multiple simulations are carried out above the outlet, with intervals of $10 \mathrm{~mm}$. The magnitude of the fluctuations may be seen based on TFI visualization in Figure 4 Multiple insonified volumes are then defined with the same volume size, the same relative positions of the acoustic source and the signal observing surfaces and with different altitudes.

In each insonified volume, a circular plane source is defined. The plane source is composed of monopole point sources on a circular plane with a diameter of $0.0254 \mathrm{~m}$ (i.e. 1 inch). The intervals of each point sources are the same as the element size of the SPECFEM mesh. Each source point emits a $1 \mathrm{MHz}$ Ricker wavelet (second derivative of a Gaussian) at the same time. The maximum amplitude of each emission is multiplied by a Hamming window function depending on the distance from the center of the circle.

Several observation surfaces are defined along the acoustic path. The receiving points where acoustic signals are recorded are placed with a $0.0005 \mathrm{~m}$ pitch. Figure 5 shows the insonified volume (central altitude $z=0.1 \mathrm{~m}$ ) and PLAJEST's 260 geometry. The point B is the origin (point $(0,0,0)$ ) of the cartesian coordinate system we used. The numbers in meters show the distance from the source to each $y$-z receiver plane. The near field limit, called Io, is calculated to be $67.17 \mathrm{~mm}$ using the empirical equation 2 , with an average temperature of 
$333.167^{\circ} \mathrm{C}\left(606.317^{\circ} \mathrm{K}\right)$, considering a transducer with a 1 inch diameter and $1 \mathrm{MHz}$ frequency. In this virtual setup, only the first two observation surfaces are in the near field. Thus, in the following analysis we mainly have acoustics virtual measurements in the far field. In the PLAJEST coordinates the limit of the near field is $x=-0.061 \mathrm{~m}$.

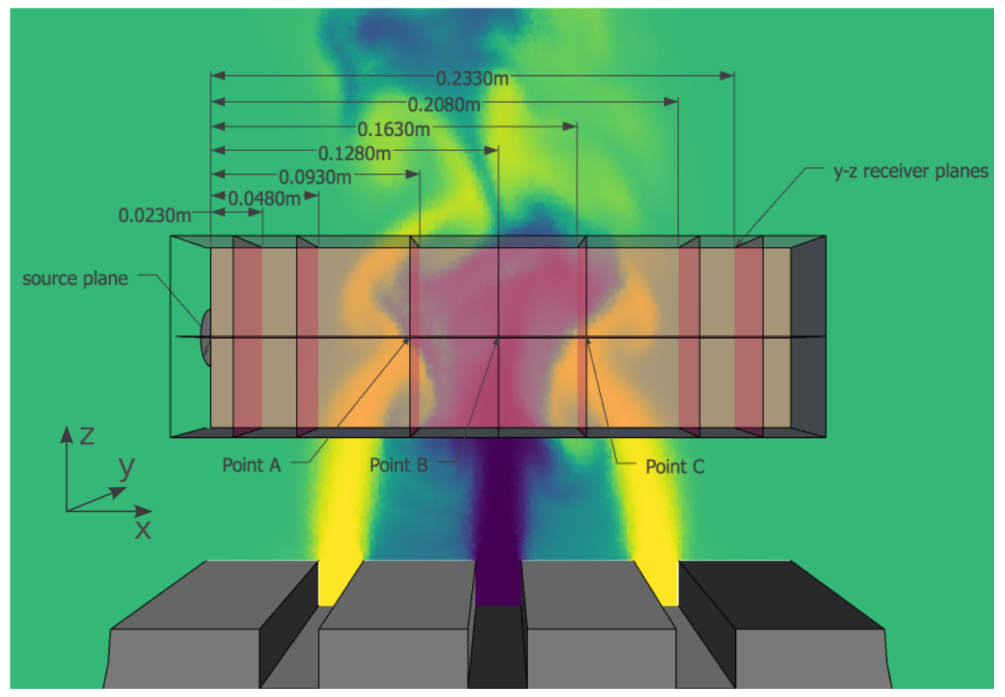

Figure 5: Relation of the positions in an insonified volume (central altitude $z=0.1 \mathrm{~m}$ ) and the geometry of PLAJEST. The points A, B and C are the positions of the reference points used for temporal analysis.

\subsection{Results of acoustic wave propagation in a single temperature field}

The figure 6 proposes an original 3D representation of the wavefront and allows to visualize 3D wave fronts based on $3 \mathrm{D}$ contour visualization. These waves are visualized from signals received in the $y-z$ receiver planes at time $=$ $200.010 \mathrm{~s}$ in the CFD simulation and at altitude $z=0.14 \mathrm{~m}$. The left image is the wave front recorded at $x=0.105 \mathrm{~m}$ and the right image is recorded at $x$ $275=-0.035 \mathrm{~m}$. Color variations represent the signal amplitude values. Blue is negative and red is positive. In order to show the inside structure of the wave front, some contour surfaces are clipped off of its half or quarter volume. The $y$ and $z$ axes correspond to the $y$ and $z$ axes of the PLAJEST's geometry. The $x$ 


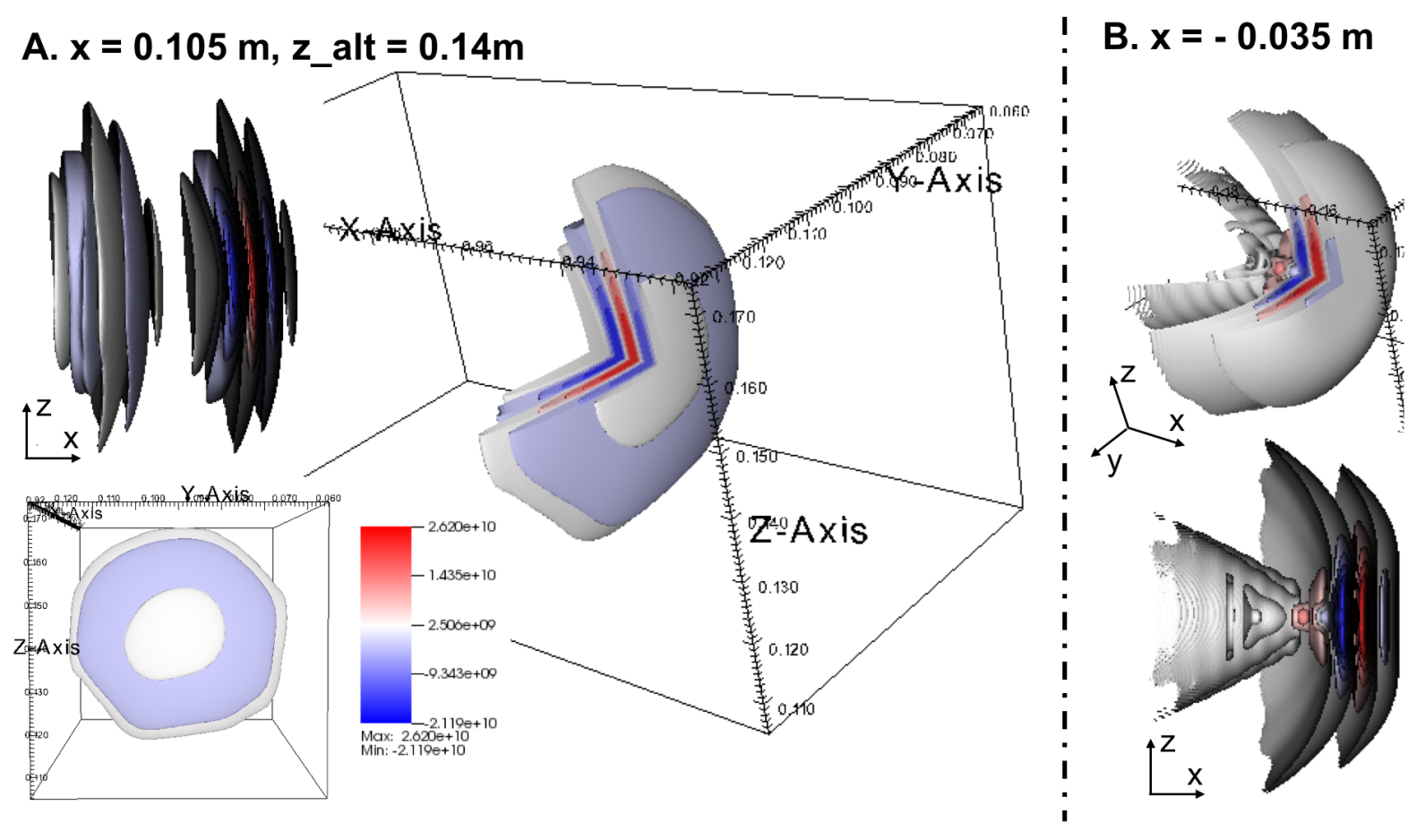

Figure 6: Visualization of the 3D wave front using contouring and clipping, at position $x=$ A) $0.105 \mathrm{~m}$ and B) $-0.035 \mathrm{~m}$ calculated at altitude $z=0.14 \mathrm{~m}$, time $=200.010 \mathrm{~s}$ of PLAJEST with a heterogeneous medium temperature. 
axis indicates time. The visual information reveals that the wave fronts having that the amount of wave deformation is not so large. Waveforms are different between waves passing from the end of the near field region to a far longer distance in the far field region (almost $4 \times$ Io, where Io is the near field limit). The wavefront in the near field has its shape that is more complex than in the far field, as expected by usual theory of wave field distribution. For this 3D visualization, we used a VTK file (Visualization Toolkit: an open-source, freely available software system for 3D computer graphics, image processing, and visualization. http://www.vtk.org) that we displayed with the visualization software VisIt.

\section{Analysis of time-varying temperature fields of PLAJEST}

\subsection{Selection of the CFD time steps}

The CFD calculation carried out by the CEA STMF has approximately $10 \mathrm{~s}$ in total, from $200.000 \mathrm{~s}$ to 210.197 with a $0.001 \mathrm{~s}$ interval. The initial $200 \mathrm{~s}$ of the calculation was dedicated to ensuring stabilization of the flow and are thus excluded in our analysis. Because of the limitation of allocated computation time on the supercomputer that we use for this work, it was not possible to run the temperature field interpolation and wave propagation calculation processes for all of these CFD time steps. Instead, we had to extract several time steps of the temperature field with a wider time interval from the CFD results. In order to select the time step interval for our acoustic simulation, we used the power spectrum density curve obtained in 28] which indicates the temperature history at $x=-0.015 \mathrm{~m}$ (between the left and center jets), $y=0.09 \mathrm{~m}$ (middle point on the $y$ axis) and $z=0.1 \mathrm{~m}$. The peak is found around $3 \mathrm{~Hz}$. Thus, to be sure to include the frequency of temperature fluctuation up to $5 \mathrm{~Hz}$, we extracted the temperature fields with a $0.1 \mathrm{~s}$ interval (i.e. $10 \mathrm{~Hz}$ ). In a first approximation

the peak was estimated to be around $2 \mathrm{~Hz}$, and in that case we could expect to 
have 5 points per period to keep the peak at $2 \mathrm{~Hz}$. This is the limit of Shannon's sampling criterion.

\subsection{Treatment of the massive amount of calculations and management of the} results

In this study, all our simulations were performed on two of the largest supercomputers in Europe: CURIE (CEA TGCC) and OCCIGEN (CINES), both part of GENCI (Grand Équipement National de Calcul Intensif). The computation domain was divided into 256 parts, and parallelized calculations were carried out. The average duration for an acoustic simulation is about 26 minutes, excluding mesh generation and the interpolation processes of the temperature fields, which are done once and for all. The duration of the interpolation of a temperature field from a TrioCFD result to SPECFEM3D is approximately 20 hours using a single $\mathrm{CPU}$ for one acoustic simulation altitude of one time step. We carried out 70 time steps of interpolation of the 3D temperature field, for 22 difference altitudes, resulting in a total of 1540 acoustic simulations to perform. As a result, the total time needed for a complete simulation of wave propagation over $7 \mathrm{~s}$ of the variable thermal-hydraulic regime is approximately equal to 687

hours, i.e. 29 days. Because of the huge number of calculations, the result data cannot be conserved as a $3 \mathrm{D}$ volume data because of the limitation of allocated storage on supercomputers. Instead of storing all results in 3D, we first selected the $2 \mathrm{D}$ planes in which we will analyze the acoustic signals. We then defined time windows for each $y-z$ plane to cut the received signals depending on the arrival time of the wave front. These time-windowed signals were then gathered as a single HDF5 binary file. The HDF5 (Hierarchical Data Format) format is a standard and widely-used binary file format that has been developed to manage extremely large and complex data collections. By using it, one can access the results faster than with other standard file formats such as e.g. ASCII, json, csv, pickle (Python-friendly binary data format) etc. 

ison with acoustic simulation results

As introduced in Equation 6, we resort to an index called Temperature Fluctuation Intensity (TFI), as used in [28. Because of the very large numbers of total time steps and also the huge number of mesh nodes included in the 340 CFD calculation results, the standard way to calculate the TFI value based on this equation is not very efficient. To calculate that TFI, we thus selected and implemented another, more advanced algorithm: the online algorithm, which we will briefly describe in this section. Figure 7 shows the calculated TFI field in 3D.We find that there are two areas where the TFI value becomes high between 345 the sodium jets at altitude $z=0.08 \mathrm{~m}$ to $0.16 \mathrm{~m}$. It should also be noted that the TFI field is not symmetric with respect to the $x$ center.

The online algorithm calculates some field value from serial data sequentially and based on a single step [42. This algorithm can be required for serial data for which each step needs a large amount of computer memory and/or when the 350 number of serial data is so large that it is very expensive to perform an entire loop of calculations more than twice. We thus applied this algorithm to compute the TFI, i.e. the standard deviation of the temperature value at a given point, because computing a standard deviation implies several loop over the whole time steps, first to calculate the mean temperature, and second to calculate the difference between a temporal value and the mean value. The PLAJEST CFD data comprise 10,000 time steps of 3D volume data with 2,039,769 mesh elements. One entire loop calculation for this data takes about 10 hours. By applying the online algorithm for the calculation of the TFI, we only need to do this long loop calculation once.

The definition of standard deviation of temperature at a given position $\boldsymbol{r}$ is

$$
\sigma^{2}(\boldsymbol{r})=\frac{1}{N} \sum_{i=1}^{N}(T(\boldsymbol{r}, i)-\bar{T}(\boldsymbol{r}))^{2}
$$

where $N$ is the total number of time steps and $\bar{T}(\boldsymbol{r})=\frac{1}{N} \sum_{i=1}^{N} T(\boldsymbol{r}, i)$ is the average temperature at position $\boldsymbol{r}$. In the online algorithm, the averaged value 


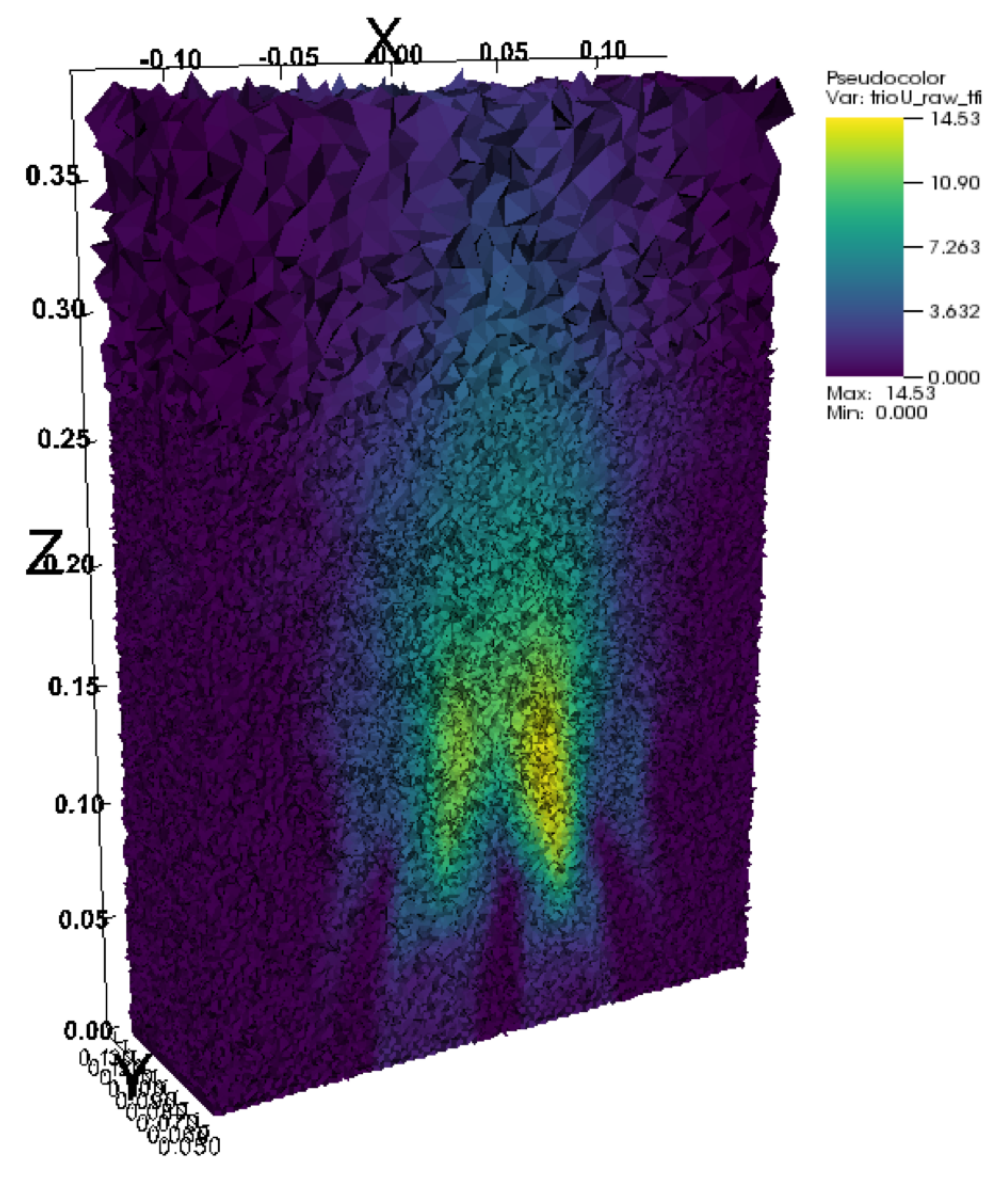

Figure 7: Visualized 3D TFI field. 


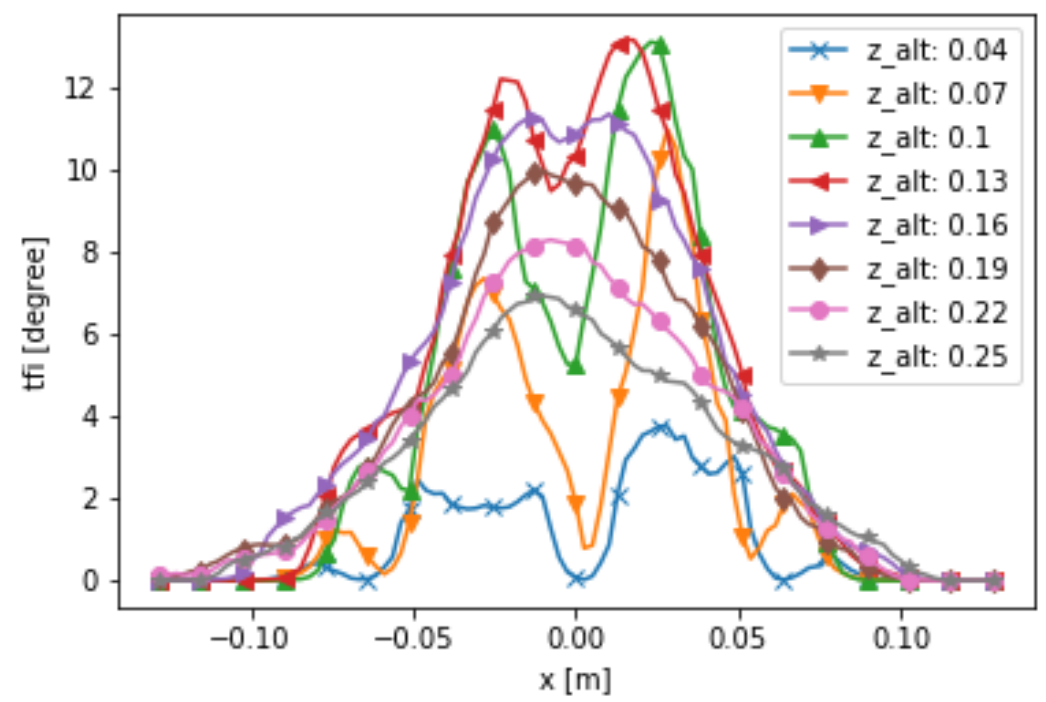

Figure 8: TFI values on the $x$ axis and at $y=0.09 \mathrm{~m}$ at several altitudes.

$\bar{T}(\boldsymbol{r}, n)$ and the term $\sum_{i=1}^{n}(T(\boldsymbol{r}, i)-\bar{T}(\boldsymbol{r}))^{2}=M_{\boldsymbol{r}, n}$ are sequentially updated for each time step during the entire loop calculation. For each time step at $n$,

$$
\begin{gathered}
\bar{T}_{\boldsymbol{r}, n}=\bar{T}_{\boldsymbol{r}, n-1}+\frac{T_{\boldsymbol{r}, n}-\bar{T}_{\boldsymbol{r}, n-1}}{n} \\
M_{\boldsymbol{r}, n}=M_{\boldsymbol{r}, n-1}+\left(T_{\boldsymbol{r}, n}-\bar{T}_{\boldsymbol{r}, n-1}\right)\left(T_{\boldsymbol{r}, n}-\bar{T}_{\boldsymbol{r}, n}\right)
\end{gathered}
$$

The standard deviation calculation is then finalized as

$$
\sigma_{r}=\sqrt{\frac{M_{r, N}}{N}} .
$$

360

Figure 8 shows the TFI values on the $x$ axis of $y=0.09 \mathrm{~m}$ at several altitudes (i.e. $z$ positions), and Figure 4 represents these TFI curves drawn in the case of the 2D TFI field. The maximum TFI value is found at around altitude $z=$ $0.13 \mathrm{~m}$. The change of shape of the curves depending on the distance from the exit of the jets seems to match with the result of [41], i.e. the curves at low altitude have two TFI peaks, and then these peaks gradually merge when the $z$ altitude increases. We will further analyze this effect in the next sections of this article. 


\subsection{Calculating the "Cumulated TFI" (CTFI) value}

The TFI calculated above is the index that evaluates the intensity of the fluctuation at one spatial point, while acoustic wave propagation will be affected not only by one position but by the whole state along its propagation path. Thus, in order to find the appropriate thermal-hydraulic index for comparison in the case of a propagating wave, we define a very new index, which we call the cumulated TFI (CTFI). The CTFI is the value that indicates the amount of TFI that the acoustic wave experiences along the central axis. We define the CTFI at the position $x_{p}$ in the propagation direction by

$$
I_{c}\left(x_{p}, z_{a l t}, R\right)=\int_{x_{s}}^{x_{p}} \int_{0}^{R} \int_{0}^{2 \pi} I\left(r, \theta, z_{a l t}\right) w(r) d \theta d r d x
$$

where $I_{c}$ is the CTFI value, $I(x, r, \theta)$ is the TFI value at $x, r, \theta$ (a graphical representation of these parameters is on Figure 10p, $x_{s}$ is the $\mathrm{x}$ coordinate of acoustic source, $z_{\text {alt }}$ is the altitude (along the $z$ axis) of the center of the acoustic source plane, $R$ is the distance from the central axis, and $w(r)$ is a weight function to make TFI values near the central axis have more effect and TFIs far from the central axis less effect. One can define several versions of the CTFI, for instance:

1. CTFI in 1D (integrated on the central $x$ axis), with $w(r)=1$ and $R=$ one mesh element size,

2. CTFI in 3D A (integrated in a domain where the acoustic beam passes), with $w(r)=1$ and $R=$ the radius of the acoustic source, i.e. $1.27 \mathrm{~cm}$,

3. CTFI in 3D B (integrated in a cylindrical volume where the acoustic beam passes), with a weighting function $w(r)=0.54+0.46 \cos \pi \frac{r}{R}$, hamming window which is the same apodization function used for simulating the acoustic plane source in this simulation, and $R=$ the radius of the acoustic source, i.e. $1.27 \mathrm{~cm}$ in our simulation.

Using a larger $w(r)$ allows us to take into account the whole ultrasonic beam. A more complex function will be needed to take into account the beam divergence. 
In this work, we only use the first definition of the CTFI, i.e. the CTFI in 1D, as a first analysis. The other possible choices may be examined in future work.

The left image on Figure 9 represents the CTFI values versus altitude $z$.

390 Each line represents the $x$ position, i.e. propagation distance (the acoustic source is placed at $x=-0.128 \mathrm{~m}$ ). The farther the $x$ position becomes, the larger the magnitude of the CTFI becomes as well. We find that the peak of the CTFI positions is around $0.13 \mathrm{~m}$ to $0.15 \mathrm{~m}$. For the $x$ position $=0.00 \mathrm{~m}$ just after the first (left side of) the high TFI zone and the middle of the central jet, the CTFI peak is slightly shifted to a higher $z$ altitude. This is caused by the slight difference of the shape and position of the high TFI zones, as one can see in Figure 5 .

The right image of Figure 9 shows the second derivatives of the CTFI curves. The second derivatives has been calculated by calculating twice the central finite differences i.e.: $f^{\prime}\left(x_{i}\right)=\frac{f\left(x_{i+1}\right)-f\left(x_{i-1}\right)}{2 h}$, where $h=x_{i+1}-x_{i}=x_{i}-x_{i-1}$. The forward/backward finite difference was used at the each end of the $x$. The inflection points are found around $z=0.08 \mathrm{~m}$ and around $z=0.18 \mathrm{~m}$ for the position $x=0.105$ (The yellow line on Figure 9). These inflection points are the same altitudes as what we defined as the merging point and combining point of 405 the standard TFI. Thus, from this result, we find that it is possible to define the merging and combining points of the TFI as the inflection points of the second derivatives of the CTFI curve. We propose thus a numerical criteria to found this specific thermal-hydraulic points.

In the following part of this work, we will study the acoustic fluctuation state based on these merging and combining points. Our objective is to propose several acoustical measurement to find the best sensitive to the behaviour of the cumulative data and so the best sensitive to find these two particular points. 

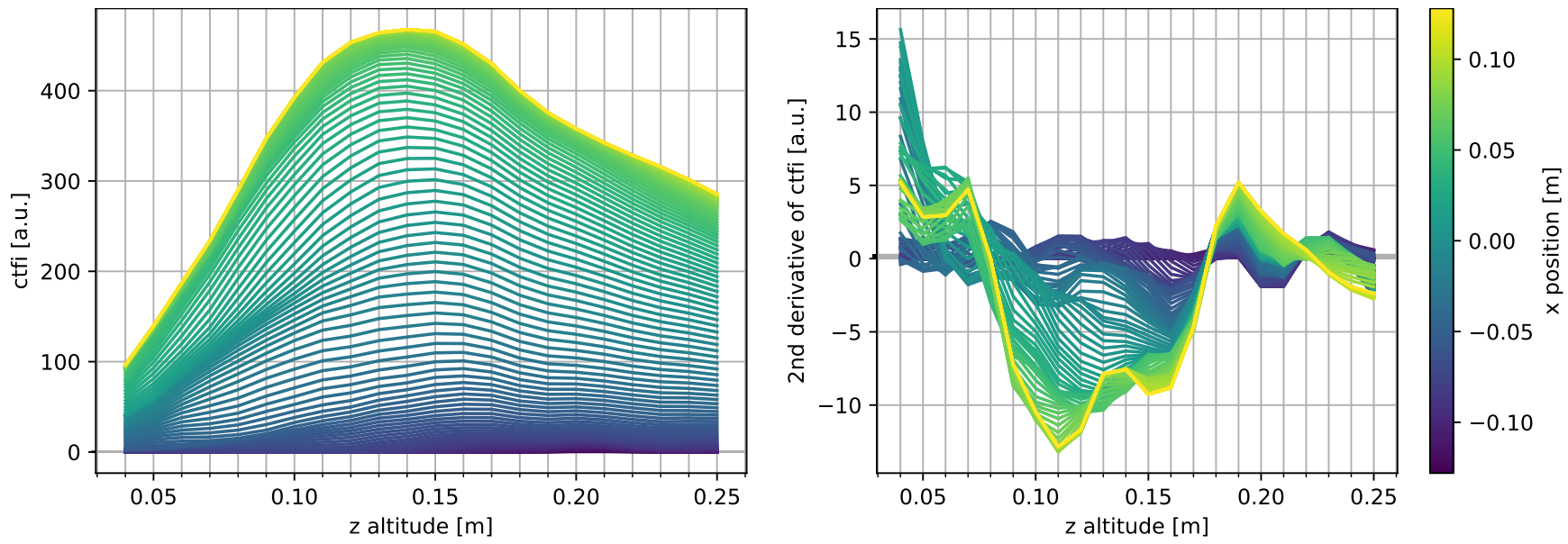

Figure 9: CTFI values on the $z$ axis at $y=0.09 \mathrm{~m}$ and second derivatives. Each line indicates the distance from the acoustic source.

\section{Comparison with acoustic simulation results}

\subsection{Fluctuation of acoustic signals}

A way to represent what we are able to measure is to consider the position of the maximum wave amplitude in a plane after the mixing of jets as indicated in figure 10. We call this point as the impact point. We define the following three acoustic indicators:

- maximum amplitude at the impact point of an acoustic path and at the center of the $y-z$ plane,

- amount of deviation $r$,

- receiving time of the maximum amplitude at the center of the $y$ - $z$ plane

Figure 10 shows the definition of the impact point and $r$. First, we investigate the transition of the deviated wave front at the farthest $y-z$ receiving plane (i.e. $x=0.105 \mathrm{~m}$ and at a distance from the source of $0.233 \mathrm{~m}$ ).

Figure 11 shows the impact points, i.e. the position where the pressure value becomes maximum in the $y$ - $z$ receiving plane. In order to show temporal changes of the positions of the impact points more clearly, the positions of the 


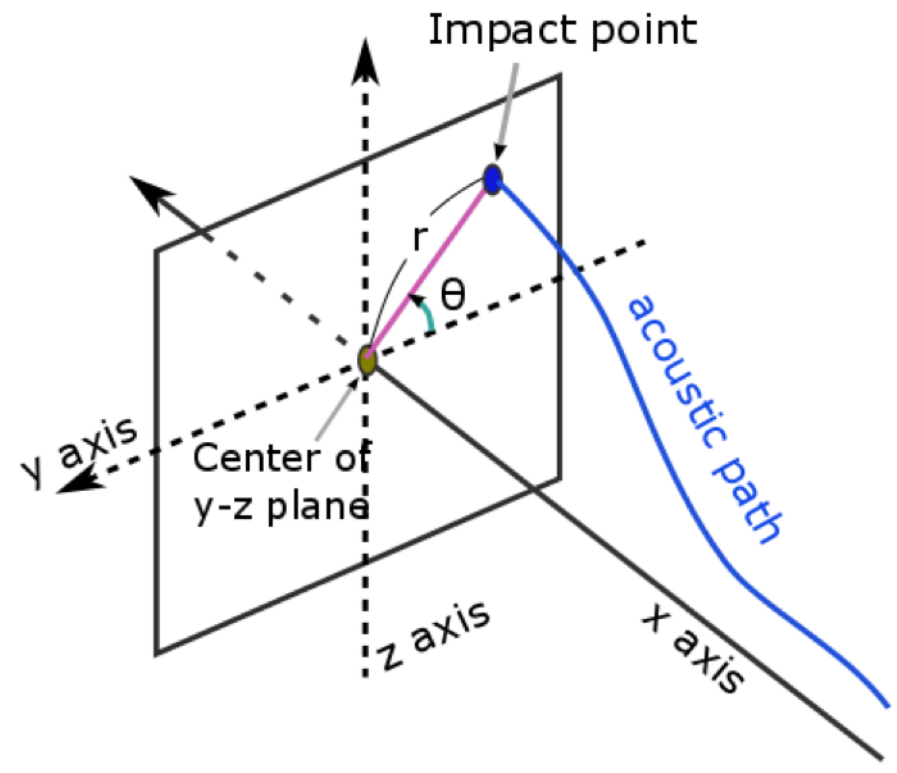

Figure 10: Definition of the impact point, of the amount of deviation $r$, and of the deviation angle $\theta$.

impact points are linked by arrows in temporal order, and digits are added to indicate the order in which the position of the impact point changes for the seven first ones. Histograms for the $y$ and $z$ axis directions are also placed, showing the mean and standard deviation values. We confirm that the standard deviation is the largest at altitude $z=0.13 \mathrm{~m}$ (number 3 of Figure 11). This is in good agreement with the peak $z$ position of the CTFI in Figure 9, At lower altitude $z=0.04 \mathrm{~m}$ and $0.09 \mathrm{~m}$, the distribution of the impact points exhibits directivity, i.e. the standard deviation for the $y$-axis direction is larger than for the $z$-axis direction. This result illustrates the fact that the $3 \mathrm{D}$ temperature fluctuation pattern before maturing of the mixing state has directivity. It is a very interesting result that indicates we can no more support classical modelling of the fluctuation of the acoustic celerity field using an isotropic Gaussian random process for the regions where the flow is still strong. From this result, we can confirm the observation by 23] of the non-applicability of an isotropic Gaussian 
random field for this region in a quantitative way.

\subsection{Standard deviation analysis}

${ }_{445}$ The left image of Figure 12 shows the standard deviation of maximum amplitudes of simulated acoustic signals at the center of the $y$ - $z$ planes for each $z$ altitude as the scatter plots and the smoothed lines calculated after a moving average triangular window with a window length of 8 . The standard deviation for the max amplitude and the other parameters were calculated from all results of 70 time steps of the acoustic propagation simulations. The right image shows the second derivatives of the average curves calculated by the same way with CTFI of Figure 9. The curves of standard deviations on the left have similar peak positions as the CTFI curves of Figure 9 and also exhibit the same position of their inflection points (right) when computing their second derivatives. Thus, the inflection points of standard deviation curves of maximum amplitudes occur at the altitudes of the merging point $(\mathrm{z}=0.08 \mathrm{~m})$ and combining point $(\mathrm{z}=$ $0.18 \mathrm{~m}$ ) of the TFI values. It is clearly a very interesting result as it proves that it is possible to find representative points of the thermal-hydraulic field using acoustic measurements.

Figure 13 is the analysis of the deviation length $r$. The left image shows the scatter plots which are the standard deviation values of $r$, and those smoothed curves calculated with the same method with the maximum amplitude. The right image shows the second derivative of these smoothed curves. The peak positions are also similar with the maximum amplitude curves, i.e. the peaks of the $x=$ $4650.035 \mathrm{~m}, 0.08 \mathrm{~m}$ and $0.105 \mathrm{~m}$ curves occur around altitude $z=0.13 \mathrm{~m}$ to $0.14 \mathrm{~m}$. Considering the inflection points of standard deviation curves of deviation length $r$ we obtain the same results as they occur at the altitudes of $(\mathrm{z}=0.08 \mathrm{~m})$ for the first one and $(\mathrm{z}=0.18 \mathrm{~m})$ for the second one. This acoustic indicator is also well adapted to find the altitudes of merging point and combining point of the thermal-hydraulic fluid.

Finally, let us analyze the simulation results based on the fluctuation of times of flight, i.e. the receiving time of the signal. We define the receiving time (the 
1) $z$ alt $0.04, x 0.105$
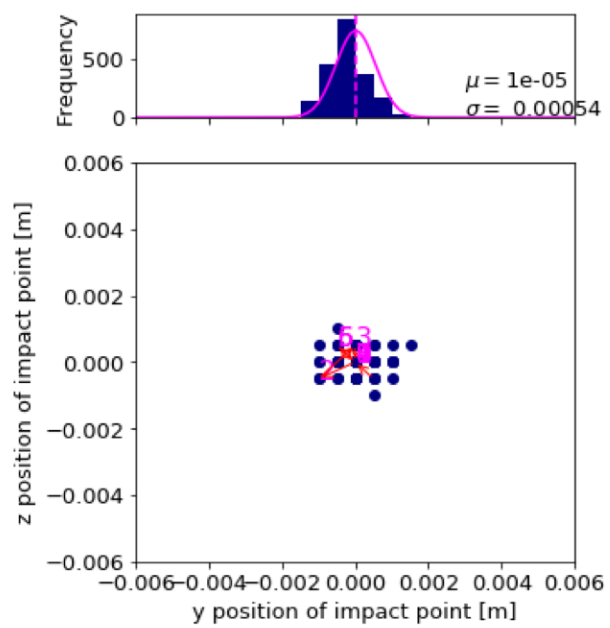

3) z alt 0.13, x 0.105
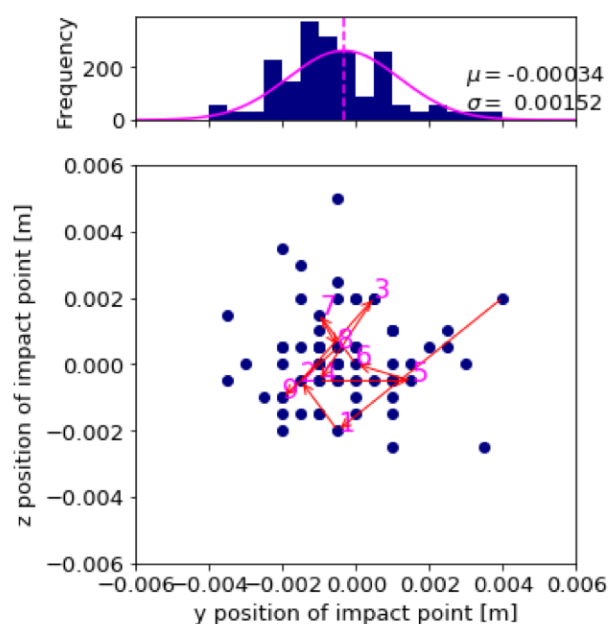

$y$ position of impact point $[\mathrm{m}]$

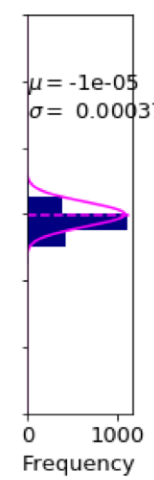

2) $z$ alt $0.09, x 0.105$
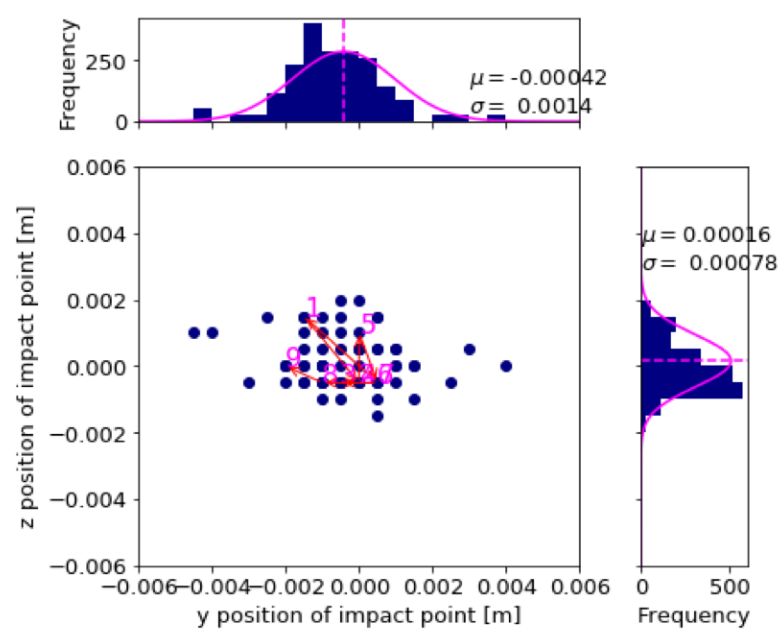

4) $z$ alt 0.21, x 0.105
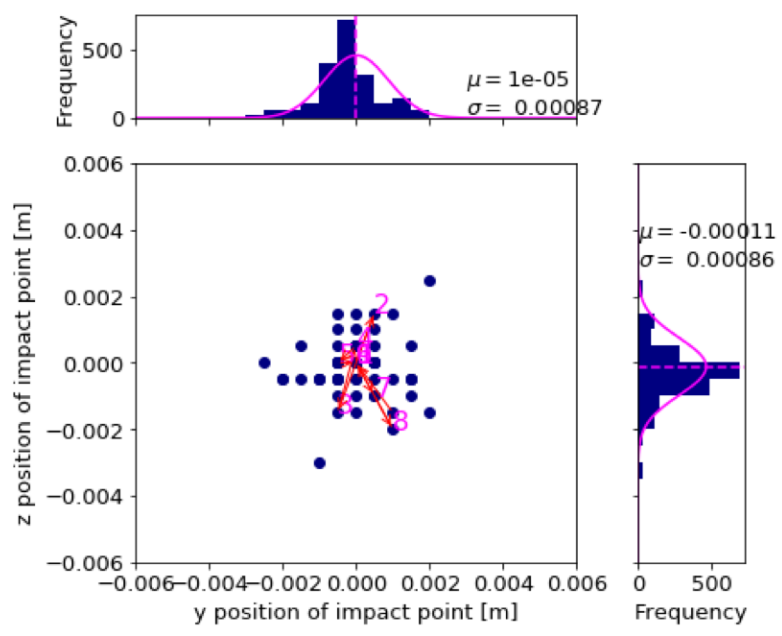

Figure 11: Movement of acoustic impact points in the $y$ - $z$ receiving plane at $x=0.105 \mathrm{~m}$, and histograms for the $y$ and $z$ directions. Movements of only the seven initial time steps are indicated with red arrows and with digits in magenta. 

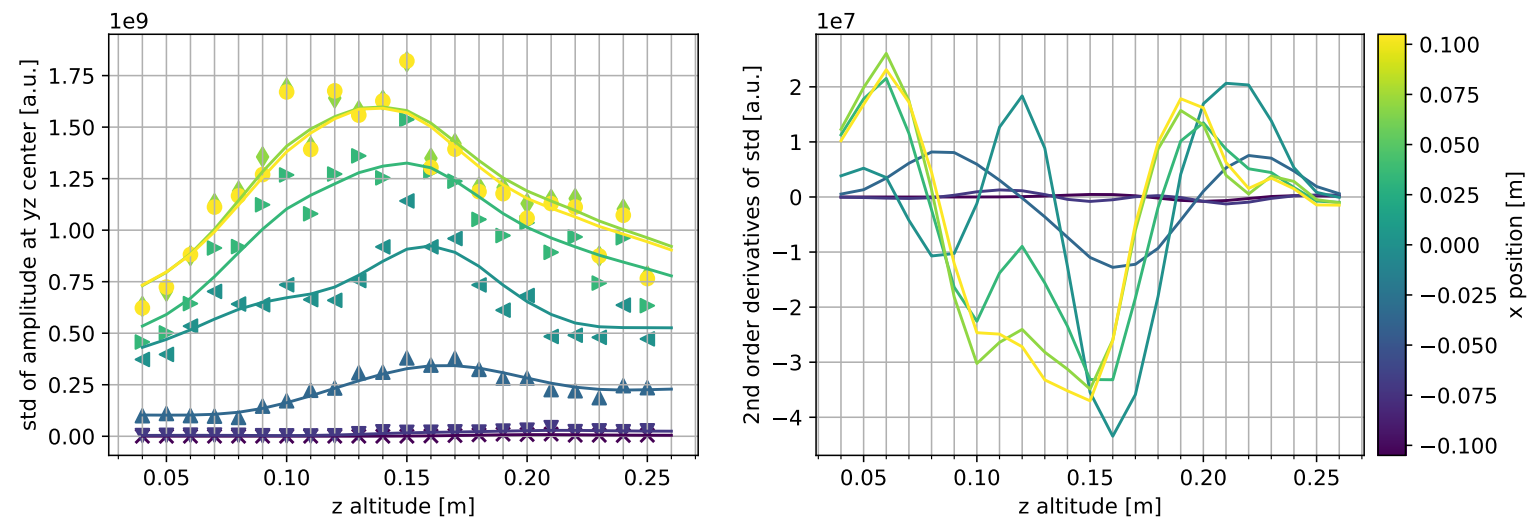

Figure 12: Left: Standard deviations of maximum amplitude at the center of each $y$ - $z$ receiving plane (scatter) and those smoothed curves with a moving average window. Right: Second derivatives of smoothed standard deviation curves.
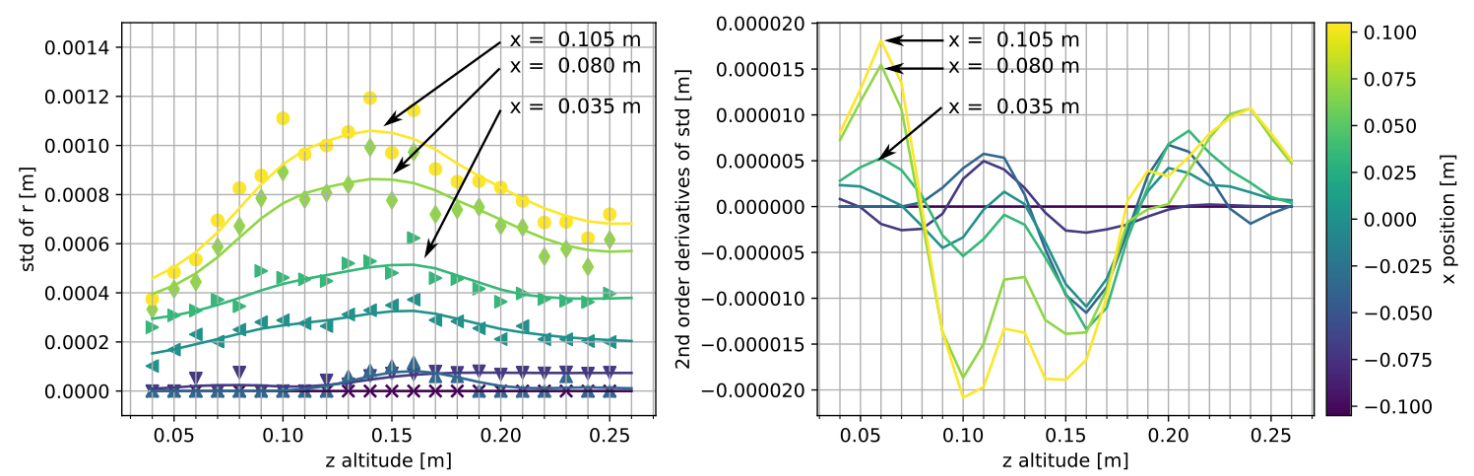

Figure 13: Left: Standard deviation of deviation length $r$ (scatter) and smoothed curves with a moving average window. Right: Second derivatives of the smoothed standard deviation lines. 
time of flight) as the peak position of the signal envelope.

Figure 14 shows the standard deviations on the scatter plots and those moving calculate the minimum TOF in this plane. Of course it will need further studies as, for a practical point of view, we should consider the resulting TOF after the integration of all 'sounds paths' on the surface of the transducer.
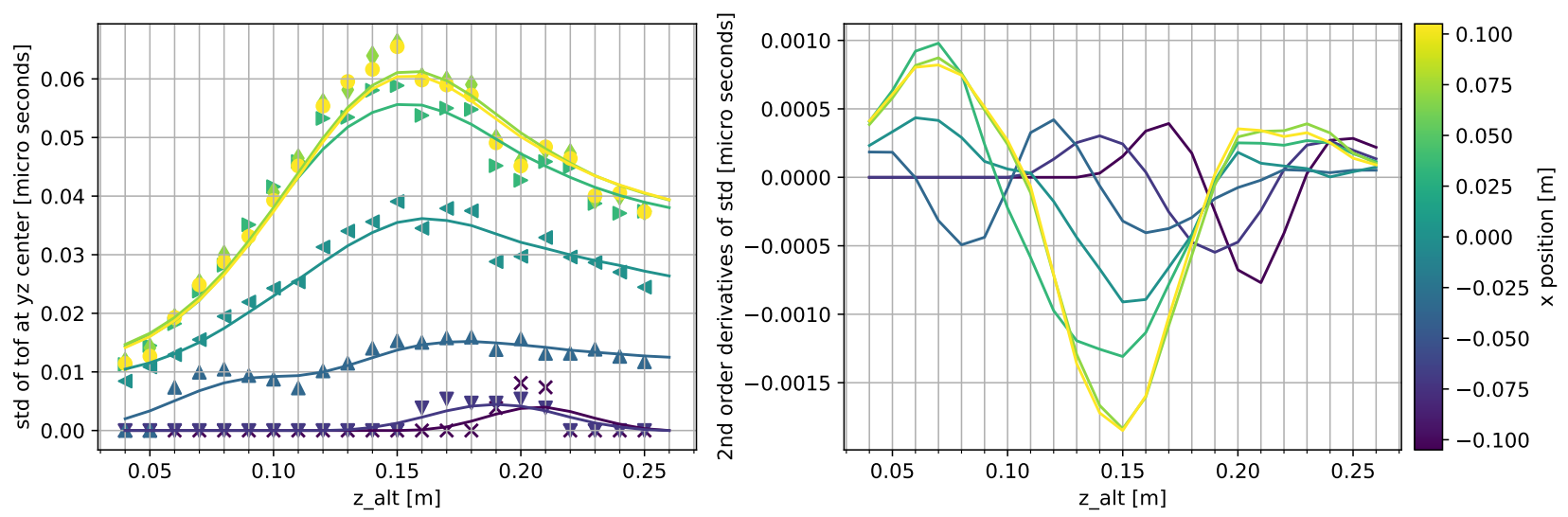

Figure 14: The standard deviation (left) and second derivative (right) of time of flight.

Figure 15 summarizes the results of the analysis and compares the standard deviation curves and second derivative curves of CTFI and the three types of acoustic indicators only at $\mathrm{x}=0.105 \mathrm{~m}$. This figure shows that the peaks of 
standard deviation curves (left) of maximum amplitude, $r$ have the same position with CTFI. However, the time of flight curve shifts about $0.01 \mathrm{~m}$ higher than the others. This shift may be seen on the inflection points of the second derivatives 495 (right) especially at the lower $\mathrm{z}$ altitudes.
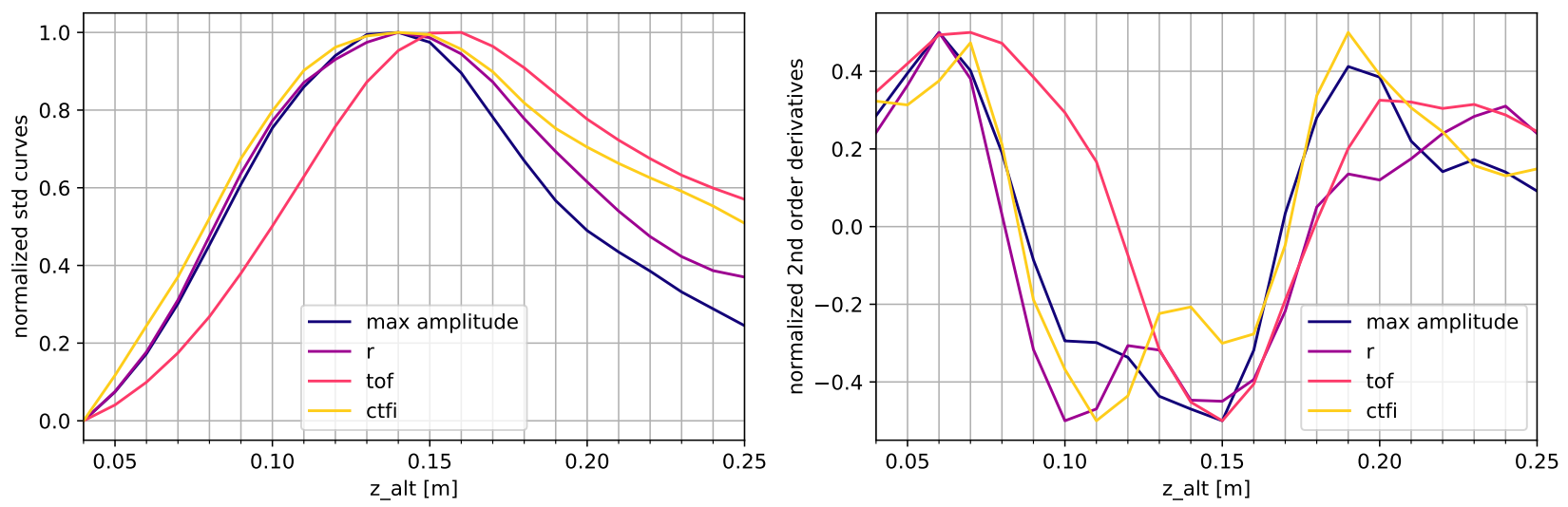

Figure 15: Comparison of the standard deviation (left) and second derivative (right) curves between max amplitude, $\mathrm{r}$, time of flight and CTFI on the $\mathrm{x}=0.105 \mathrm{~m}$ plane.

\section{Conclusions}

Developing a very strong numerical scheme, with huge computation power, we studied the effect of a realistic heterogeneous temperature field on wave propagation in four dimensions (i.e., three spatial dimensions and time). We first simulated wave propagation at a fixed instant of a Plajest's CFD simulation, changing the altitude of the volume scanned by transducer, to analyse variations caused by the flows of liquid metal on wave propagation. To quantify the effect of the 3D temperature fied fluctuations on an acoustic path we defined a new measurement index, called CTFI (Cumulated Temperature Fluctuation Intensity). We exhibited specific altitude ranges, corresponding to the merging of jets, at which a strong effect may occur on acoustic wave propagation. We thus defined two altitudes at which the state of temperature fluctuation changes: one is the merging point, where the two high TFI zones between each of three 
jets start to merge, and the other is the combining point of the TFI, where the merging of the TFI zones is completed. We demonstrate that these altitude can be detected as inflection points of the second derivative of the CTFI curves.

We then carried out the same acoustic simulation but for multiple CFD time steps in order to investigate the relation between acoustic measurements and the thermal-hydraulic state. We calculated the standard deviations of several acoustic indicators (maximum amplitude, amount of deviation $r$, and time of flight) to study the fluctuation of the acoustic signals and to correlate with the thermal field fluctuations. As a first result of our standard deviation analysis, we found that at the low altitude where the mixing of flows is not enough matured, the temperature heterogeneity may cause directive fluctuation of acoustic propagation. This means that the application of an isotropic Gaussian random field to simulate temperature field is not an efficient solution as the fluctuating values generated by an isotropic Gaussian random field always have a normal distribution and so produces no directivity.

We also found that the second derivatives of the standard deviation of acoustic indicators have inflection points at altitudes close to those at which we defined the merging and combining points. The results for the maximum amplitude and the amount of deviation $r$ are better than for time of flight. The $r$ parameter will require an array of transducers to be measured with high resolution. At this moment the recommended parameter would the the maximum value. Of course a combination of the two parameters will increase the confidence in the measure.

In the future it will be possible to increase the validation of the use of acoustic measurements to monitor complex thermal-hydraulic fields by developing spectral analysis to characterize the temporal fluctuation of the fluid. It would require to refine the time-step between two acoustic simulations.

\section{Acknowledgements}

This study was granted access to the French HPC resources of TGCC/CINES under allocations 2016107626, 2016047165 and 2016047748 made by GENCI. We 
thank also Antoine Gershenfeld and Pierre Emmanuel Angéli, from CEA / DES / IRESNE / DM2S / STMF, for sharing their thermal-hydraulic data of Plajest and their advises to analyse these data. This work was developed within the framework of the MISTRAL joint research laboratory between Aix-Marseille University, CNRS, Centrale Marseille and CEA.

\section{References}

[1] GIF, Framework agreement for international collaboration on research and development of Generation-IV nuclear energy system, Stationery Office, 2005.

[2] GIF, Technology roadmap update for Generation IV Nuclear Energy Systems, January 2014 (2014).

[3] P. Hora, O. Červená, Determination of Lamb wave dispersion curves by means of Fourier transform, Applied and Computational Mechanics 6 (2012) $5-16$.

[4] F. Baqué, F. Reverdy, J.-M. Augem, J. Sibilo, Development of tools, instrumentation and codes for improving periodic examination and repair of SFRs, Science and Technology of Nuclear Installations 2012 (2012) 718034. doi:10.1155/2012/718034.

[5] F. Baqué, O. Gastaldi, F. Jadot, F. Le Bourdais, J. Sibilo, J. M. Augem, ASTRID in service inspection and repair: Review of r\&d program and associated results, in: S. Monti (Ed.), Proceedings of the Fast Reactors and Related Fuel Cycles: Safe Technologies and Sustainable Scenarios (FR13) conference, International Atomic Energy Agency (IAEA), Paris, France, 2015, pp. IAEA-CN-199/33.

[6] J. L. Berton, G. Loyer, Continuous monitoring of the position of two subassemblies heads of Phenix at 350 MWth power and $550 \mathrm{deg} \mathrm{C}$ temperature, in: SMORN VII, Nuclear Energy Agency of the OECD (NEA), 1996, p. 578. 
[7] B. Lu, M. Darmon, C. Potel, V. Zernov, Models comparison for the scattering of an acoustic wave on immersed targets, in: Journal of Physics: Conference Series, Vol. 353, IOP Publishing, 2012, p. 012009.

[8] B. Lü, M. Darmon, L. Fradkin, C. Potel, Numerical comparison of acoustic wedge models, with application to ultrasonic telemetry, Ultrasonics 65 (2016) $5-9$.

[9] P. Y. Ufimtsev, Fundamentals of the physical theory of diffraction, John Wiley \& Sons, 2014.

[10] M.-A. Ploix, G. Corneloup, J. Moysan, J.-P. Jeannot, Investigation of ultrasonic backward energy from various edges as a function of their $2 \mathrm{~d} / 3 \mathrm{~d}$ geometry and of the incidence angle, for application to ultrasonic thermometry at the outlet of a tube, Case Studies in Nondestructive Testing and Evaluation 6 (2016) 38-44.

[11] J. A. McKnight, I. D. Macleod, E. J. Burton, Remote temperature measurement, uS Patent 4,655,992 (1987).

[12] N. Massacret, J. Moysan, M. A. Ploix, J. P. Jeannot, G. Corneloup, Simplified modeling of liquid sodium medium with temperature and velocity gradient using real thermal-hydraulic data: Application to ultrasonic thermometry in Sodium Fast Reactor, AIP Conference Proceedings 1511 (1) (2013) 1693-1700. doi:10.1063/1.4789245.

[13] N. Massacret, étude d'une méthode ultrasonore d'estimation des températures locales du sodium liquide en sortie coeur RNR-Na, Ph.D. thesis, Aix-Marseille University, France (2014).

[14] N. Massacret, J. Moysan, M. A. Ploix, J. P. Jeannot, G. Corneloup, Modelling of ultrasonic propagation in turbulent liquid sodium with temperature gradient, Journal of Applied Physics 115 (20) (2014) 204905. doi:10.1063/1.4875876. 
[15] M. Cavaro, C. Payan, J. Moysan, F. Baqué, Microbubble cloud characterization by nonlinear frequency mixing, J. Acoust. Soc. Am. 129 (5) (2011) 179-183. doi:10.1121/1.3565474

[16] L. D'Hondt, M. Cavaro, C. Payan, S. Mensah, Acoustical characterisation and monitoring of microbubble clouds, Ultrasonics 96 (2019) 10-17. doi: $10.1016 /$ j.ultras.2019.03.009.

[17] D. Haubensack, Calculs thermohydrauliques du collecteur chaud de la chaudière ASTRID au nominal en soutien l'instrumentation coeur CFVBD-16/10 et ISIR (État d'avancement fin 2016), Tech. rep., Commissariat à l'énergie atomique et aux énergies alternatives (CEA), Direction de l'énergie nucléaire, France (2016).

[18] E. Tarpara, V. Patankar, N. Vijayan Varier, Under-sodium ultrasonic viewing for fast breeder reactors: A review, Tech. rep., BARC, BHABHA ATOMIC RESEARCH CENTRE, MUMBAI, INDIA, bARC/2016/E/014 (2016).

[19] G. Chaitanya, A. Kumar, Detection of subassembly bowing in a fast breeder reactor using glancing angle ultrasonic imaging, Annals of Nuclear Energy 150 (2021) 107821. doi:https://doi.org/10.1016/j.anucene.2020. 107821

[20] V. Sobolev, Database of thermophysical properties of liquid metal coolants for GEN-IV, Tech. rep., SCK-CEN, Mol, Belgium, BLG-1069 (2011).

[21] D. Fiorina, Application of the Gaussian beam summation method to the study of the ultrasonic wave propagation in a turbulent medium, Ph.D. thesis, École Centrale de Lyon, France (1998).

[22] B. Iooss, P. Blanc-Benon, C. Lhuillier, Statistical moments of travel times at second order in isotropic and anisotropic random media, Waves in Random Media 10 (3) (2000) 381. 
[26] N. Kimura, H. Miyakoshi, H. Ogawa, H. Kamide, Y. Miyake, K. Nagasawa, Study on convective mixing phenomena in parallel triple-jet along wall Comparison of temperature fluctuation characteristics between sodium and water, in: Proceedings of the NURETH-11 conference, Oct. 2-6, 2005, no. 22 in 37052929, Avignon, France, 2005, p. 1.

[27] N. Kimura, H. Kamide, P. Emonot, K. Nagasawa, Study on thermal striping phenomena in triple-parallel jet - Transfer characteristics of temperature fluctuation in sodium and water based on conjugated numerical simulation, in: Proceedings of the NUTHOS-7 conference, Oct. 5-9, 2008, BB2008115136001509, Seoul, Korea, 2008, p. 4802.

[28] P. E. Angeli, Large-eddy simulation of thermal striping in WAJECO and PLAJEST experiments with TrioCFD, in: Proceedings of 16th International Topical Meeting on Nuclear Reactor Thermal Hydraulics (NURETH-16), 2015, pp. 1223-1236.

[29] H. Childs, E. Brugger, B. Whitlock, J. Meredith, S. Ahern, D. Pugmire, K. Biagas, M. Miller, C. Harrison, G. H. Weber, H. Krishnan, T. Fogal, 
A. Sanderson, C. Garth, E. W. Bethel, D. Camp, O. Rubel, M. Durant, J. M.

655

[31] M. Nagaso, D. Komatitsch, J. Moysan, C. Lhuillier, Wave propagation simulation in the upper core of sodium-cooled fast reactors using a spectralelement method for heterogeneous media, in: EPJ Web of Conferences, Vol. 170, EDP Sciences, 2018, p. 03006.

660

[34] D. Komatitsch, D. Michéa, G. Erlebacher, Porting a high-order finite-element earthquake modeling application to NVIDIA graphics cards using CUDA, Journal of Parallel and Distributed Computing 69 (5) (2009) 451-460. doi:10.1016/j.jpdc.2009.01.006

[35] D. Komatitsch, G. Erlebacher, D. Göddeke, D. Michéa, High-order finiteelement seismic wave propagation modeling with MPI on a large GPU

口 cluster, J. Comput. Phys. 229 (20) (2010) 7692-7714. doi:10.1016/j.jcp. 2010.06 .024 
[36] P. J. Davis, P. Rabinowitz, Methods of numerical integration (Computer science and applied mathematics), Academic Press, New York, USA, 1984.

[37] C. Canuto, M. Y. Hussaini, A. Quarteroni, T. A. Zang, Spectral methods, Springer, Berlin, Germany, 2011.

[38] A. Durve, A. W. Patwarddhan, I. Banarjee, G. Padmakumar, G. Vaidyanathan, Numerical investigation of mixing in parallel jets, Nuclear

(1) Engineering and Design 242 (2012) 78-90. doi:10.1016/j.nucengdes

685 2011.10.051.

[39] B. Zang, T. New, On the wake-like vortical arrangement and behaviour associated with twin jets in close proximity, Experimental Thermal and Fluid Science 69 (2015) 127-140. doi:10.1016/j.expthermflusci.2015. 08.004

690

[40] S. Ghahremanian, K. Svensson, M. J. Tummers, B. Moshfegh, Near-field mixing of jets issuing from an array of round nozzles, International Journal of

口 Heat and Fluid Flow 47 (2014) 84-100. doi:10.1016/j.ijheatfluidflow. 2014.01.007.

[41] A. Durve, A. W. Patwardhan, I. Banarjee, G. Padmakumar, 695

प G. Vaidyanathan, Thermal striping in triple jet flow, Nuclear Engineering and Design 240 (10) (2010) 3421-3434. doi:10.1016/j.nucengdes. 2010 07.025

[42] D. E. Knuth, The Art of Computer Programming, Vol. 1, Fundamental Algorithms, 3rd Edition, Addison-Wesley Professional, 1997. 